\title{
Combined grip strength and calf circumference as a useful prognostic system in patients with liver diseases: a large cohort study
}

\author{
Hiroki Nishikawa $^{1,2 \#}$, Kazunori Yoh ${ }^{1 \#}$, Hirayuki Enomoto ${ }^{1}$, Takashi Nishimura ${ }^{1}$, Shuhei Nishiguchi ${ }^{3}$, \\ Hiroko Iijima $^{1}$
}

${ }^{1}$ Department of Internal Medicine, Division of Gastroenterology and Hepatology, Hyogo College of Medicine, Nishinomiya, Hyogo, Japan; ${ }^{2}$ Center for Clinical Research and Education, Hyogo College of Medicine, Nishinomiya, Hyogo, Japan; Kano General Hospital, Osaka, Osaka, Japan

Contributions: (I) Conception and design: H Nishikawa; (II) Administrative support: H lijima, S Nishiguchi; (III) Provision of study materials or patients: All authors; (IV) Collection and assembly of data: All authors; (V) Data analysis and interpretation: H Nishikawa, K Yoh, H Enomoto; (VI) Manuscript writing: All authors; (VII) Final approval of manuscript: All authors.

\#These authors contributed equally to this work.

Correspondence to: Hiroki Nishikawa, MD, PhD. Department of Internal Medicine, Division of gastroenterology and hepatology, Hyogo College of Medicine, Nishinomiya, Hyogo. 1-1, Mukogawacho, Nishinomiyashi, Hyogo 663-8501, Japan. Email: nishikawa_6392_0207@yahoo.co.jp.

Background: Sarcopenia and body composition can be associated with mortality in chronic liver diseases (CLDs). We sought to identify predictors in CLD patients ( $\mathrm{n}=631,309$ males) and create a prognostic model using easily available indexes.

Methods: Reference values for low-grip strength (GS) were $26 \mathrm{~kg}$ in men and $18 \mathrm{~kg}$ in women. Reference values for low-skeletal muscle index (SMI) were $7.0 \mathrm{~kg} / \mathrm{m}^{2}$ in men and $5.7 \mathrm{~kg} / \mathrm{m}^{2}$ in women using bioelectrical impedance analysis (BIA). Reference values for low-calf circumference (CC) were $34 \mathrm{~cm}$ in men and $33 \mathrm{~cm}$ in women. Reference values for high-waist circumference were $85 \mathrm{~cm}$ in men and $90 \mathrm{~cm}$ in women. Using significant factors in the multivariate analysis contributing to the overall survival (OS), we created a simple predictive model. Akaike information criterion (AIC) was compared.

Results: Men $(\mathrm{P}<0.0001)$, presence of liver cirrhosis $(\mathrm{LC})(\mathrm{P}<0.0001)$, presence of hepatocellular carcinoma (HCC) $(\mathrm{P}<0.0001)$, low-GS $(\mathrm{P}<0.0001)$, low-CC $(\mathrm{P}<0.0001)$, serum albumin $(\mathrm{P}=0.0355)$, estimated glomerular filtration rate $(\mathrm{P}=0.0461)$, hepatitis $\mathrm{B}$ virus $(\mathrm{P}=0.0044)$ and hepatitis $\mathrm{C}$ virus $(\mathrm{P}<0.0001)$ were significant factors contributing to the OS by the multivariate analysis. The study subjects were classified into the 4 groups (combined GS-SMI system): (I) low-GS and low-SMI (sarcopenia, n=73); (II) low-GS and highSMI ( $\mathrm{n}=65)$; (III) high-GS and low-SMI ( $\mathrm{n}=110)$; and (IV) high-GS and high-SMI ( $\mathrm{n}=383)$. The cumulative OS rates were well stratified among 4 groups (overall $\mathrm{P}<0.0001$, AIC $=360.895$ ). The study subjects were also classified into the 4 groups (combined GS-CC system): (I) low-GS and low-CC (n=60); (II) low-GS and high-CC ( $\mathrm{n}=78)$; (III) high-GS and low-CC ( $\mathrm{n}=70)$; and (IV) high-GS and high-CC ( $\mathrm{n}=423)$. The cumulative OS rates were also well stratified among 4 groups (overall $\mathrm{P}<0.0001, \mathrm{AIC}=349.521$ ). In receiver operating characteristic (ROC) curve analysis for CC based on the OS, the optimal cutoff point in men was $34.6 \mathrm{~cm}$ [area under the ROC $(\mathrm{AUC})=0.70$, sensitivity $=0.558$, specificity $=0.842$ ], and that in women was $32.8 \mathrm{~cm}$ (AUC $=0.72$, sensitivity $=0.619$, specificity $=0.787)$.

Conclusions: CC can be an alternative marker for muscle mass in CLD patients. Our proposed combined GS$\mathrm{CC}$ system can be helpful in the community settings without special equipment for muscle mass measurement.

Keywords: Chronic liver disease (CLD); grip strength (GS); skeletal muscle mass; calf circumference (CC); predictive model

Submitted Oct 14, 2020. Accepted for publication Jan 25, 2021.

doi: 10.21037/atm-20-6901

View this article at: http://dx.doi.org/10.21037/atm-20-6901

(c) Annals of Translational Medicine. All rights reserved. 


\section{Introduction}

More than three decades have passed since Rosenberg's proposal of sarcopenia in elderly people in 1989 (1). Sarcopenia, which is characterized by skeletal mass loss and muscle strength loss and/or physical activity decline, is frequently encountered in chronic liver diseases (CLDs) because of the disease burden itself as well as aging (2-6). CLD patients with sarcopenia can involve both impaired protein synthesis and accelerated muscle proteolysis in skeletal muscle $(5,6)$. Sarcopenia in CLDs has been recently gaining much research interest due to its prognostic significance (3,7-10). A previous meta-analysis reported the close association between sarcopenia and mortality in CLD patients (3). However, it is controversial as to which of muscle strength or muscle mass is a stronger predictor in CLD patients. For the past 2 or 3 decades, sarcopenia researches have mainly focused on muscle mass (11). While in our recent study, we emphasized the significance of grip strength (GS) on composite hepatic events in CLD patients (12). Hanai et al. reported that reduced GS rather than skeletal muscle mass or fat mass was significantly linked to an increased risk of mortality in cirrhotic patients (13). GS well reflects muscle strength for the entire body, and also reflects nutritional status as muscle function responses earlier to starvation status (14-16). A previous large observational study showed that measuring of GS is a simple and cost-effective method for risk stratification of prognosis (17). Additionally, an increase of GS significantly contributed to a reduced risk of cancer-related death (18).

How to employ convenient markers in the daily clinical practice is essential from the perspective of suppressing medical costs. Body composition measurement is suitable for nutritional assessment in routine clinical settings because it is simple and minimally invasive (19-21). In particular, calf circumference (CC) is recommended to measure in the revised Asian Working Group for Sarcopenia (AWGS) guidelines on the earlier detection of sarcopenic persons (22). While the measurement of waist circumference (WC) is used for the assessment of metabolic syndrome (23). However, prognostic impacts of anthropometric measurements in CLD patients are unclear.

A prognostic model using simple indexes that can be used by anyone is very useful in the daily medical care. It is particularly significant in facilities without advanced medical equipment. In this study, we sought to identify prognostic markers for CLD patients, and also create a prognostic model as a useful tool for clinicians. We present the following article in accordance with the STROBE reporting checklist (available at http://dx.doi.org/10.21037/ atm-20-6901).

\section{Methods}

\section{Patients}

Using a retrospective computerized database, 631 CLD individuals who visited our hospital between January 2013 and April 2020 were collected. CLD was defined as a condition in which hepatitis was confirmed to persist for more than 6 months. Data for muscle strength (i.e., GS) and muscle mass using bioelectrical impedance analysis (BIA), and data for body composition (CC and WC) at baseline (i.e., at the beginning of the follow-up) were collected. Diagnosis for liver cirrhosis (LC) and hepatocellular carcinoma (HCC) were determined according to the current guidelines $(24,25)$. The most suitable interventional strategy for each underlying liver disease was performed (24,26,27). HCC therapy was based on the current guidelines $(25,28,29)$. No patient received liver transplantation in the follow-up period. Patients with far advanced HCC, other advanced malignancies, severe heart failure, severe ascites or severe malnutrition were not included. The study was conducted in accordance with the Declaration of Helsinki (as revised in 2013) with ethical approval from the institutional review board in our hospital (approval number 3469). An opt out method was employed considering the retrospective nature of this study.

\section{$G S, S M I, C C$ and $W C$}

Reference values for low-GS were $26 \mathrm{~kg}$ in men and $18 \mathrm{~kg}$ in women based on the Japanese Society of Hepatology (JSH) guidelines (30). Likewise, reference values for lowskeletal muscle index [SMI, SMI indicates appendicular muscle mass divided by height squared $\left(\mathrm{kg} / \mathrm{m}^{2}\right)$ ] were $7.0 \mathrm{~kg} / \mathrm{m}^{2}$ in men and $5.7 \mathrm{~kg} / \mathrm{m}^{2}$ in women using BIA (30). BIA is currently the standard method for the assessment of skeletal muscle mass in CLDs (29). Reference values for low-CC were $34 \mathrm{~cm}$ in men and $33 \mathrm{~cm}$ in women based on the AWGS guidelines (22,31). Reference values for high-WC were $85 \mathrm{~cm}$ in men and $90 \mathrm{~cm}$ in women based on the Japanese criteria for visceral fat obesity (23). All of anthropometric measurements were done by an expert nutritionist. 


\section{Primary outcome measure and study design of our study}

The primary outcome measure was the overall survival (OS, all-cause mortality). Study subjects were followed from the anthropometric measurement at baseline until death or the last follow-up visit. First, factors contributing to the OS were identified using univariate and multivariate analyses. Second, using sarcopenia-related variables or body compositionrelated variables, prognostic systems were created. Predictability in each prognostic model was assessed.

\section{Statistics}

In the univariate analyses of items contributing to the OS, the median value for each item was selected for the classification of our cohort. Factors with a $\mathrm{P}$ value $<0.05$ (univariate analysis) were entered into the multivariate Cox hazard model. Survival curves were created by the Kaplan-Meier method with the log-rank test. Akaike information criterion (AIC) in each prognostic system was tested (32). The smaller the AIC value, the better the model was considered (32). In the analysis of correlation between parameters, Pearson's correlation coefficient (r) was employed. Receiver operating characteristic curve (ROC) analysis was done for estimating the area under the ROC (AUC) for CC with the optimal cutoff value determined by Youden index (33). A $\mathrm{P}<0.05$ denotes statistical significance [statistical analysis software: JMP 14 (SAS Institute Inc., Cary, NC, USA)].

\section{Results}

\section{Patient backgrounds}

Our patient backgrounds ( $\mathrm{n}=631,309$ males) were presented in Table 1. The median (interquartile range, IQR) age was $65(52.0,71)$ years. The median follow-up interval was 3.53 years. LC was seen in 226 patients (35.8\%: Child-Pugh $\mathrm{A} / \mathrm{B} / \mathrm{C}$ in $166 / 53 / 7$ patients, respectively). Forty-nine men $(15.9 \%)$ had a GS decrease, while 89 women $(27.6 \%)$ had a GS decrease (30). Seventy-six men (24.6\%) had an SMI decrease, while 107 women (33.2\%) had an SMI decrease (30). Forty-nine men (15.9\%) had a CC decrease, while 81 women (25.2\%) had a CC decrease (22). Two hundred and six men (66.7\%) had a WC increase, while 103 women (32.0\%) had a WC increase (23).

\section{Causes of death and cumulative OS rates for all cases}

During the observation period, 64 patients $(10.1 \%)$ succumbed: liver failure-related death in 28 patients, HCC progression-related death in 17 and other causes in 19. The 1-, 3- and 5-year cumulative survival rates for all cases were $97.5 \%, 91.2 \%$ and $85.8 \%$ (Figure 1 ).

\section{Uni- and multivariate analyses of factors contributing to the OS}

The univariate analysis of factors associated with the OS identified that 13 factors were significant: age $\geq 65$ years $(\mathrm{P}<0.0001)$, gender $(\mathrm{P}=0.0020)$, liver disease etiology $(\mathrm{P}=0.0002)$, low-GS $(\mathrm{P}<0.0001)$, low-SMI $(\mathrm{P}=0.0002)$, low$\mathrm{CC}(\mathrm{P}<0.0001)$, presence of $\mathrm{LC}(\mathrm{P}<0.0001)$, presence of HCC $(\mathrm{P}<0.0001)$, aspartate aminotransferase $>27 \mathrm{IU} / \mathrm{L}(\mathrm{P}=0.0055)$, serum albumin $\geq 4.2 \mathrm{~g} / \mathrm{dL}(\mathrm{P}<0.0001)$, prothrombin time $\geq 92.1 \%(\mathrm{P}=0.0031)$, platelet count $\geq 17.8 \times 10^{4} / \mathrm{mm}^{3}$ $(\mathrm{P}=0.0014)$ and estimated glomerular filtration rate (eGFR) $>81 \mathrm{~mL} / \mathrm{min} / 1.73 \mathrm{~m}^{2}(\mathrm{P}=0.0433)$ (Table 2). Multivariate analysis of the 13 factors identified that men $(\mathrm{P}<0.0001)$, presence of LC $(\mathrm{P}<0.0001)$, presence of HCC $(\mathrm{P}<0.0001)$, low-GS $(\mathrm{P}<0.0001)$, low-CC $(\mathrm{P}<0.0001)$, serum albumin $\geq 4.2 \mathrm{~g} / \mathrm{dL}(\mathrm{P}=0.0355)$, eGFR $>81 \mathrm{~mL} / \mathrm{min} / 1.73 \mathrm{~m}^{2}$ $(\mathrm{P}=0.0461)$, hepatitis $\mathrm{B}$ virus $(\mathrm{P}=0.0044)$ and hepatitis $\mathrm{C}$ virus $(\mathrm{P}<0.0001)$ were significant factors contributing to the $\mathrm{OS}$ (Table 3). Hazard ratio and $95 \%$ confidence interval for each variable were demonstrated in Table 3.

\section{Cumulative OS rates in combined GS and SMI, and in combined GS and CC}

Muscle strength and muscle mass are well established and validated prognostic markers $(12,22,30)$. Thus, the study subjects were classified into the 4 groups (combined GSSMI system): (I) subjects with low-GS and low-SMI (type A, sarcopenia, $n=73$ ); (II) subjects with low-GS and highSMI (type B, n=65); (III) subjects with high-GS and lowSMI (type C, n=110); and (IV) subjects with high-GS and high-SMI (type D, n=383). AIC value on OS was calculated. The OS rates were well stratified among 4 groups (overall $\mathrm{P}<0.0001$, AIC $=360.895$ ) (Figure 2A).

Next, considering the results of multivariate analysis, the study subjects were also classified into the 4 groups (combined GS-CC system): (I) subjects with low-GS and low-CC (type E, n=60); (II) subjects with low-GS and high-CC (type F, n=78); (III) subjects with highGS and low-CC (type G, n=70); and (IV) subjects with high-GS and high-CC (type $\mathrm{H}, \mathrm{n}=423$ ). The OS rates were well stratified among 4 groups (overall $\mathrm{P}<0.0001$, 
Table 1 Patient backgrounds ( $\mathrm{n}=631)$

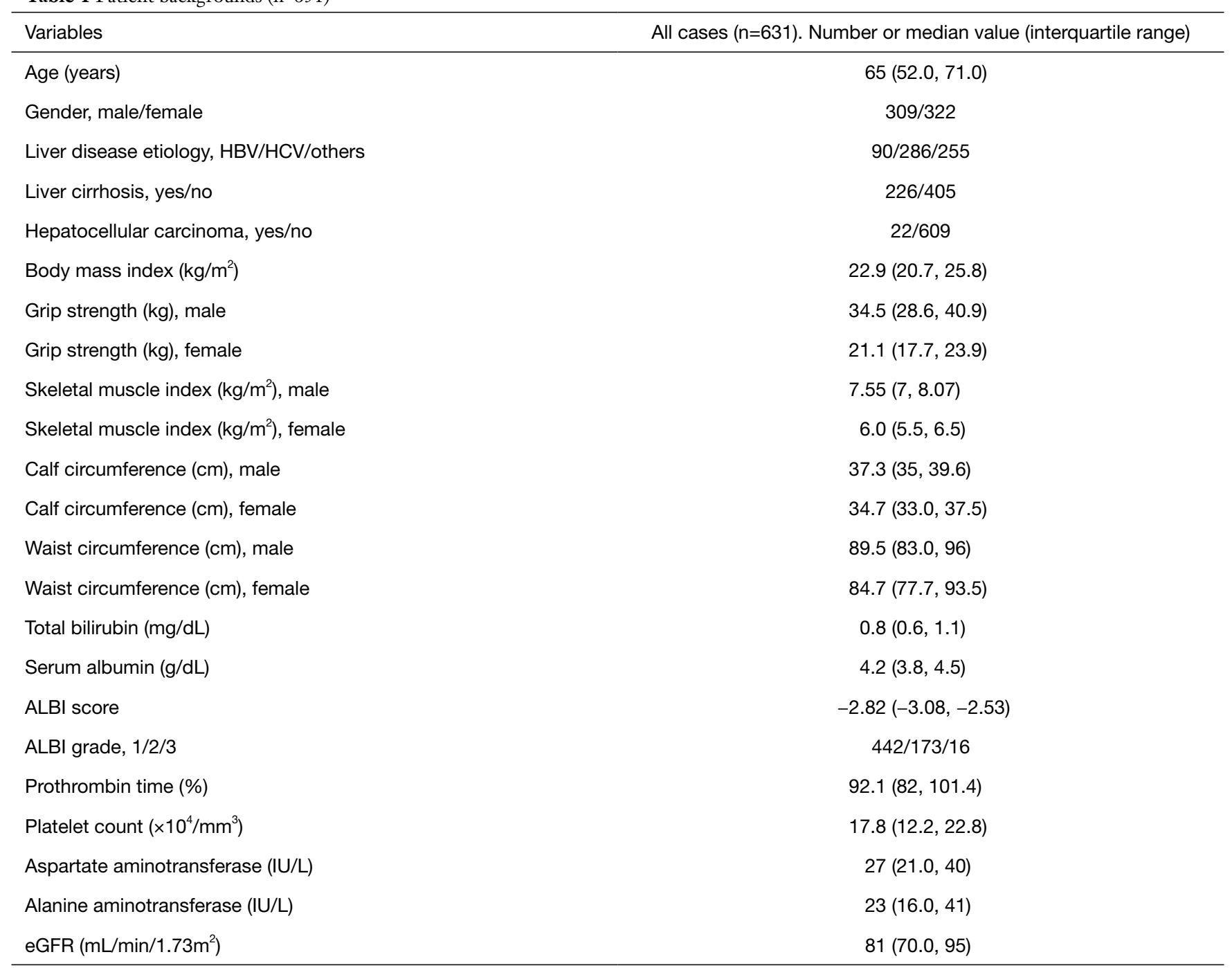

HBV, hepatitis B virus; HCV, hepatitis C virus; ALBI, albumin-bilirubin; eGFR, estimated glomerular filtration rate.

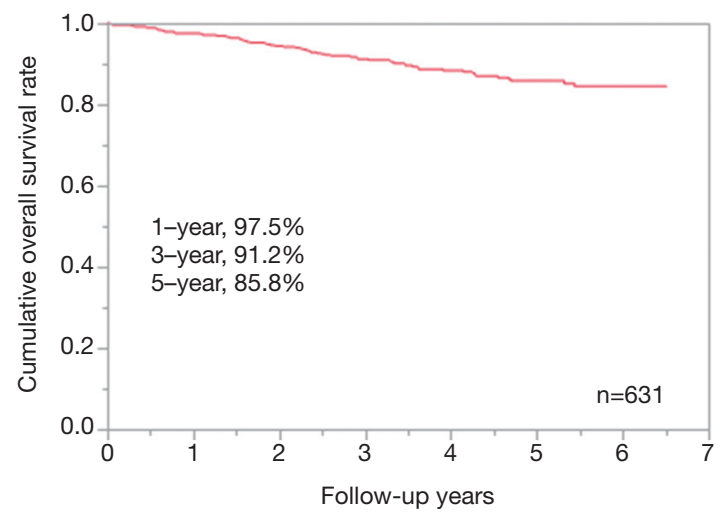

Figure 1 Mortality rates for all cases $(\mathrm{n}=631)$.
AIC $=349.521)$ (Figure 2B).

\section{Subgroup analysis according to gender (combined} GS-SMI system vs. combined GS-CC system)

To confirm the validity of our proposed combined GSCC system, we performed subgroup analyses (four sections below "Cumulative OS rates in combined GS and SMI, and in combined GS and CC"). In men ( $\mathrm{n}=309)$, the OS rates were well stratified by GS and SMI [type A $(n=24)$, type B $(\mathrm{n}=25)$, type $\mathrm{C}(\mathrm{n}=52)$ and type $\mathrm{D}(\mathrm{n}=208)]$ (overall $\mathrm{P}<0.0001$, $\mathrm{AIC}=251.335$ ). (Figure $3 A$ ) Likewise, the $\mathrm{OS}$ rates were well stratified by GS and CC [type E ( $n=21)$, type F ( $n=28)$, type $G$ 
Table 2 Univariate analyses of variables contributing to overall survival ( $\mathrm{n}=631)$

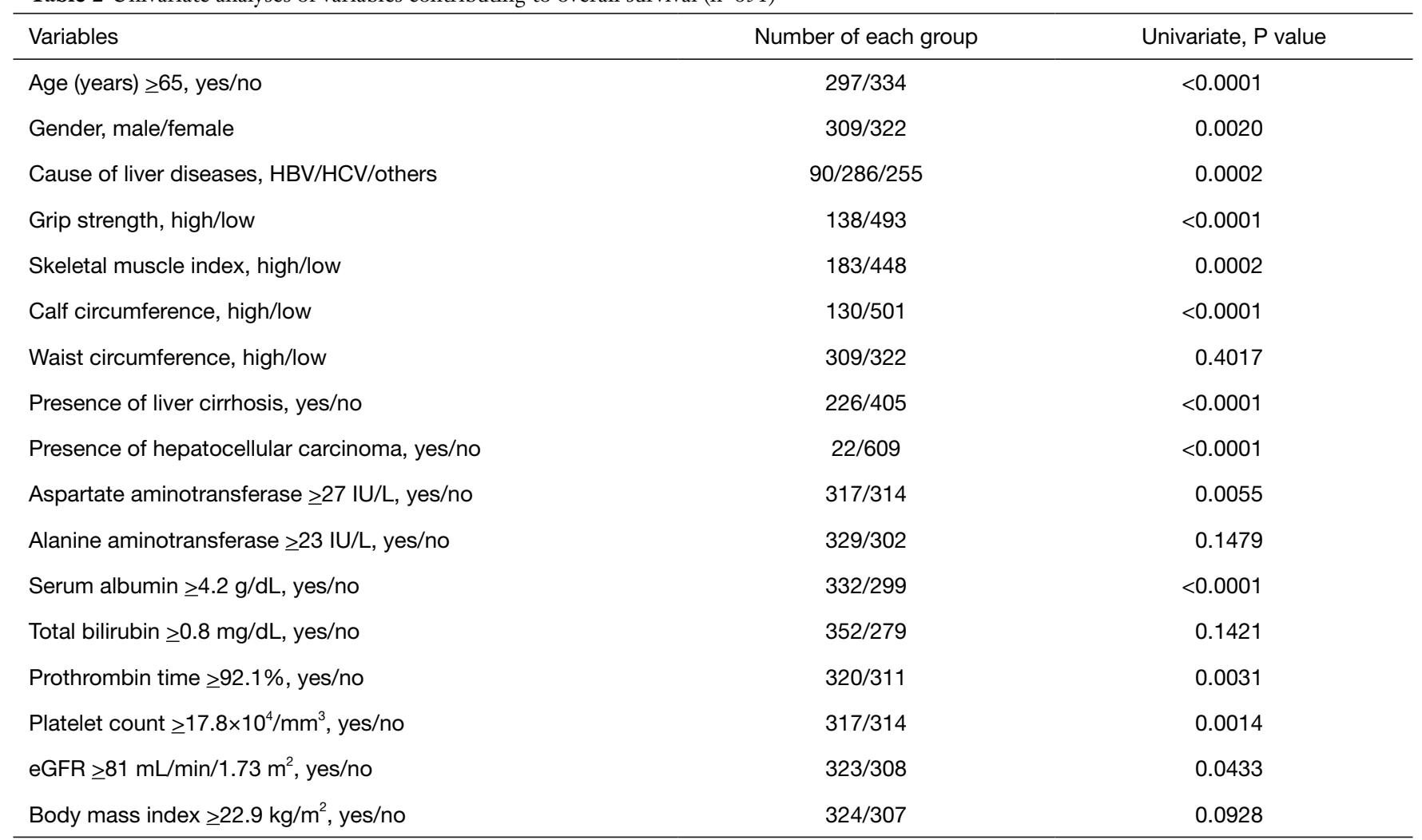

HBV, hepatitis B virus; HCV, hepatitis C virus; eGFR, estimated glomerular filtration rate.

$(\mathrm{n}=28)$ and type $\mathrm{H}(\mathrm{n}=232)]$ (overall $\mathrm{P}<0.0001, \mathrm{AIC}=190.833)$ (Figure 3B).

In women $(\mathrm{n}=322)$, the OS rates were well stratified by GS and SMI [type A (n=49), type B $(n=40)$, type C $(n=58)$ and type $\mathrm{D}(\mathrm{n}=175)]$ (overall $\mathrm{P}=0.0011$, AIC $=157.273$ ) (Figure 3C). Similarly, the OS rates were well stratified by GS and CC [type $E(n=39)$, type $F(n=50)$, type $G(n=42)$ and type $\mathrm{H}(\mathrm{n}=191)$ ] (overall $\mathrm{P}<0.0001$, AIC $=137.698)$ (Figure 3D).

\section{Subgroup analysis according to age (combined GS-SMI system $v s$. combined GS-CC system)}

In patients over 65 years $(\mathrm{n}=297)$, the OS rates were well stratified by GS and SMI [type A ( $n=59)$, type B $(n=47)$, type $\mathrm{C}(\mathrm{n}=65)$ and type $\mathrm{D}(\mathrm{n}=126)]$ (overall $\mathrm{P}<0.0001$, $\mathrm{AIC}=239.657$ ) (Figure 4A). Likewise, the OS rates were well stratified by GS and CC [type E ( $n=50)$, type $F(n=56)$, type $\mathrm{G}(\mathrm{n}=46)$ and type $\mathrm{H}(\mathrm{n}=145)]($ overall $\mathrm{P}<0.0001$, $\mathrm{AIC}=235.501)$ (Figure 4B).

In patients less than 65 years $(\mathrm{n}=334)$, the OS rates were well stratified by GS and SMI [type A ( $n=14)$, type B ( $n=18)$, type $\mathrm{C}(\mathrm{n}=45)$ and type $\mathrm{D}(\mathrm{n}=257)$ ] (overall $\mathrm{P}<0.0001$, $\mathrm{AIC}=122.593$ ) (Figure 4C). Similarly, the OS rates were well stratified by GS and CC [type $\mathrm{E}(\mathrm{n}=10)$, type $\mathrm{F}(\mathrm{n}=22)$, type $\mathrm{G}(\mathrm{n}=24)$ and type $\mathrm{H}(\mathrm{n}=278)$ ] (overall $\mathrm{P}<0.0001$, $\mathrm{AIC}=116.241)$ (Figure $4 D$ ).

Subgroup analysis according to the LC status (combined GS-SMI system $v \boldsymbol{s}$. combined GS-CC system)

In patients with LC $(n=226)$, the OS rates were well stratified by GS and SMI [type A ( $n=33)$, type B $(n=40)$, type $\mathrm{C}(\mathrm{n}=43)$ and type $\mathrm{D}(\mathrm{n}=110)$ ] (overall $\mathrm{P}<0.0001$, AIC =217.659). (Figure 5A) Likewise, the OS rates were well stratified by GS and CC [type $E(n=31)$, type $F(n=42)$, type $\mathrm{G}(\mathrm{n}=31)$ and type $\mathrm{H}(\mathrm{n}=122)$ ] (overall $\mathrm{P}<0.0001$, $\mathrm{AIC}=210.434)$ (Figure 5B).

In patients with non-LC $(\mathrm{n}=405)$, the OS rates were well stratified by GS and SMI [type A $(n=40)$, type B $(n=25)$, type $\mathrm{C}(\mathrm{n}=67)$ and type $\mathrm{D}(\mathrm{n}=273)]($ overall $\mathrm{P}<0.0001$, AIC =104.64). (Figure 5C) Similarly, the OS rates were well stratified by GS and CC [type E (n=29), type F ( $n=36)$, type $\mathrm{G}(\mathrm{n}=39)$ and type $\mathrm{H}(\mathrm{n}=301)$ ] (overall $\mathrm{P}<0.0001$, 
Table 3 Multivariate analyses of variables contributing to overall survival ( $\mathrm{n}=631)$

\begin{tabular}{|c|c|c|c|}
\hline Variables & \multicolumn{3}{|c|}{ Multivariate analysis } \\
\hline Age $\geq 65$ years & 1.177 & $0.585-2.298$ & 0.6400 \\
\hline Male & 3.464 & $1.938-6.362$ & $<0.0001$ \\
\hline Presence of liver cirrhosis & 6.041 & $2.467-15.266$ & $<0.0001$ \\
\hline Low-grip strength & 6.110 & $3.393-11.213$ & $<0.0001$ \\
\hline Low-calf circumference & 5.031 & $2.621-9.835$ & $<0.0001$ \\
\hline Low-skeletal muscle index & 1.435 & $0.712-2.917$ & 0.3136 \\
\hline Serum albumin $\geq 4.2 \mathrm{~g} / \mathrm{dL}$ & 0.474 & $0.227-0.951$ & 0.0355 \\
\hline Aspartate aminotransferase $\geq 27 \mathrm{IU} / \mathrm{L}$ & 1.180 & $0.633-2.234$ & 0.6039 \\
\hline eGFR $\geq 81 \mathrm{~mL} / \mathrm{min} / 1.73 \mathrm{~m}^{2}$ & 0.553 & $0.305-0.990$ & 0.0461 \\
\hline \multicolumn{4}{|l|}{ Cause of liver disease } \\
\hline HBV-related & 0.297 & $0.111-0.698$ & 0.0044 \\
\hline HCV-related & 0.184 & $0.098-0.335$ & $<0.0001$ \\
\hline Others & & Reference & \\
\hline
\end{tabular}

eGFR, estimated glomerular filtration rate; HBV, hepatitis B virus; HCV, hepatitis C virus.

$\mathrm{AIC}=107.584)($ Figure $5 D)$.

\section{Subgroup analysis in patients without HCC at baseline (combined GS-SMI system vs. combined GS-CC system)}

In non-HCC patients at baseline ( $\mathrm{n}=609)$, the OS rates were well stratified by GS and SMI [type A ( $n=67)$, type B $(n=59)$, type $\mathrm{C}(\mathrm{n}=107)$ and type $\mathrm{D}(\mathrm{n}=376)]$ (overall $\mathrm{P}<0.0001$, $\mathrm{AIC}=286.784$ ) (Figure 6A). Likewise, the OS rates were well stratified by GS and CC [type $\mathrm{E}(\mathrm{n}=56)$, type $\mathrm{F}(\mathrm{n}=70)$, type $\mathrm{G}(\mathrm{n}=67)$ and type $\mathrm{H}(\mathrm{n}=416)]$ (overall $\mathrm{P}<0.0001$, AIC $=272.938)$. Due to the small number of HCC cases $(\mathrm{n}=22)$, we did not perform subgroup analysis in HCC patients (Figure 6B).

\section{Correlation between CC and SMI according to gender}

The association in CC and SMI was investigated using Pearson's correlation coefficient (r). In men, significant correlation between CC and SMI was found ( $\mathrm{r}=0.80$, $\mathrm{P}<0.0001$ ) (Figure 7 A). Similarly, in women, significant correlation between CC and SMI was found $(r=0.86$, $\mathrm{P}<0.0001)$ (Figure $7 B$ ).

\section{Cutoff values of CC in male and female based on the OS}

The AWGS guidelines set the cutoff values of CC $34 \mathrm{~cm}$ in men and $33 \mathrm{~cm}$ in women (23). To confirm the validity of these cutoff points in AWGS, ROC analyses were performed. In ROC analysis for the cutoff value of CC based on the OS, the optimal cutoff point in men was $34.6 \mathrm{~cm}$ (AUC $=0.70$, sensitivity $=0.558$, specificity $=0.842)$, and that in women was $32.8 \mathrm{~cm}$ (AUC $=0.72$, sensitivity $=0.619$, specificity $=0.787)($ Figure $8 A, B)$.

\section{Discussion}

As mentioned earlier, a prognostic model using easily available indexes appears to be very helpful in the daily medical care. In this study, we primarily aimed to create a simple prognostic system both in community settings and hospital settings. Especially in facilities that cannot 


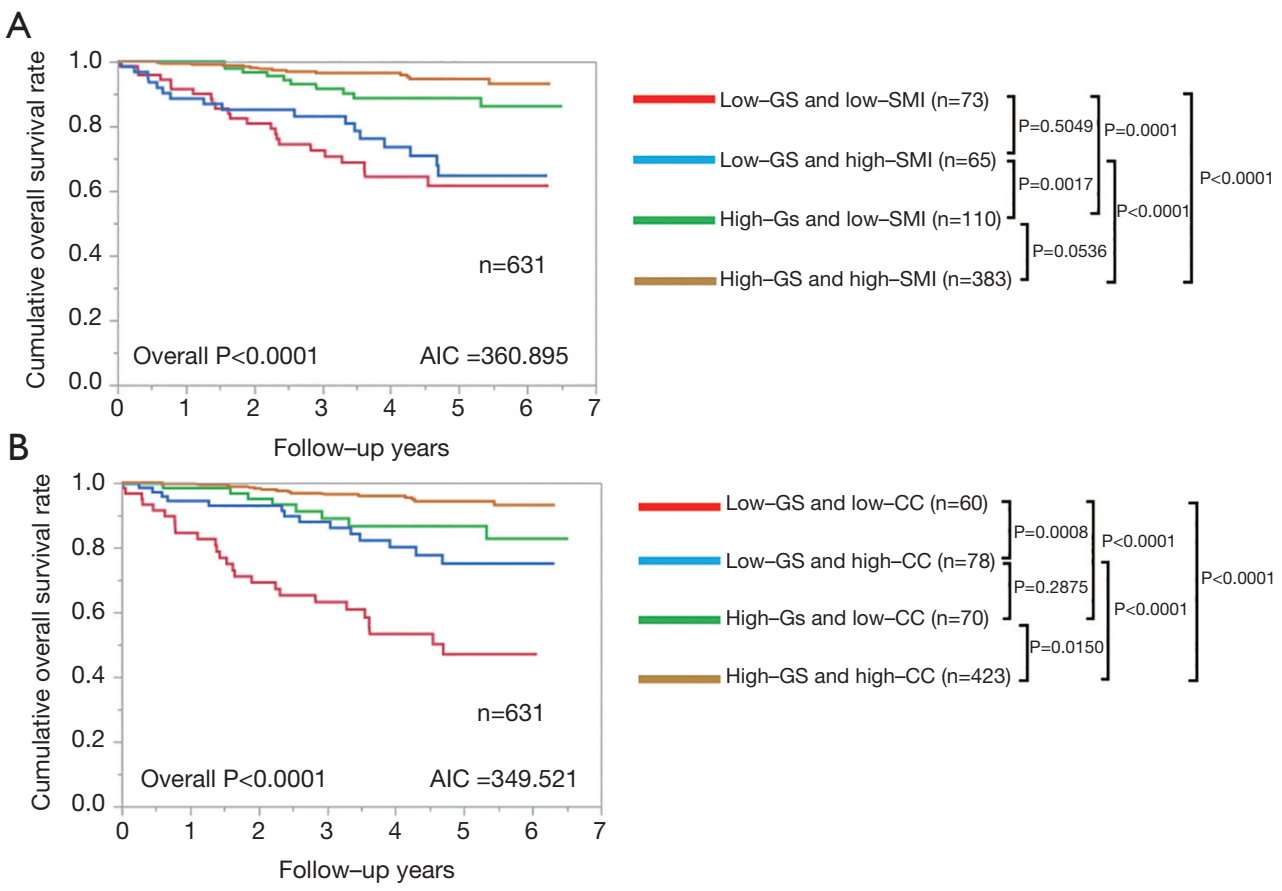

Figure 2 Mortality rates stratified by GS and SMI or CC for all cases. (A) Mortality rates stratified by GS and SMI for all cases. (B) Mortality rates stratified by GS and CC for all cases. GS, grip strength; SMI, skeletal muscle index; CC, calf circumference.

A

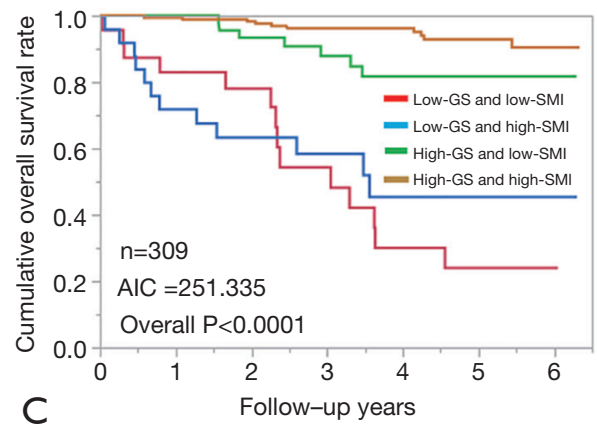

C

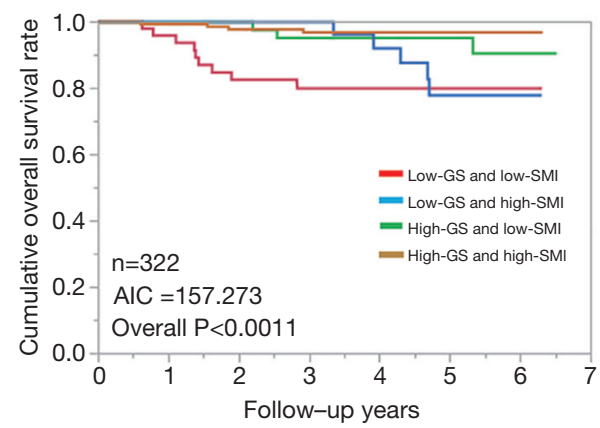

B
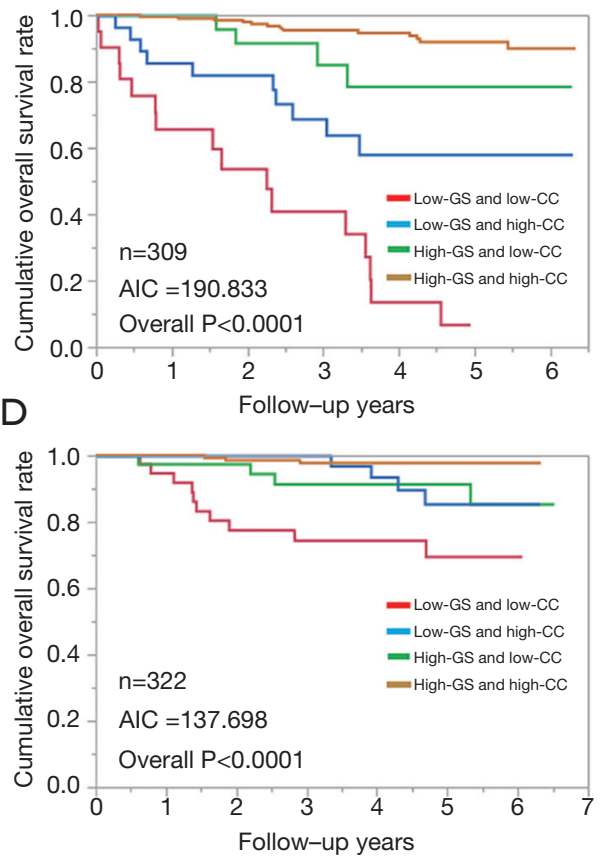

Figure 3 Mortality rates stratified by GS and SMI or CC according to gender. (A) Mortality rates stratified by GS and SMI in men. (B) Mortality rates stratified by GS and CC in men. (C) Mortality rates stratified by GS and SMI in women. (D) Mortality rates stratified by GS and CC in women. GS, grip strength; SMI, skeletal muscle index; CC, calf circumference. 

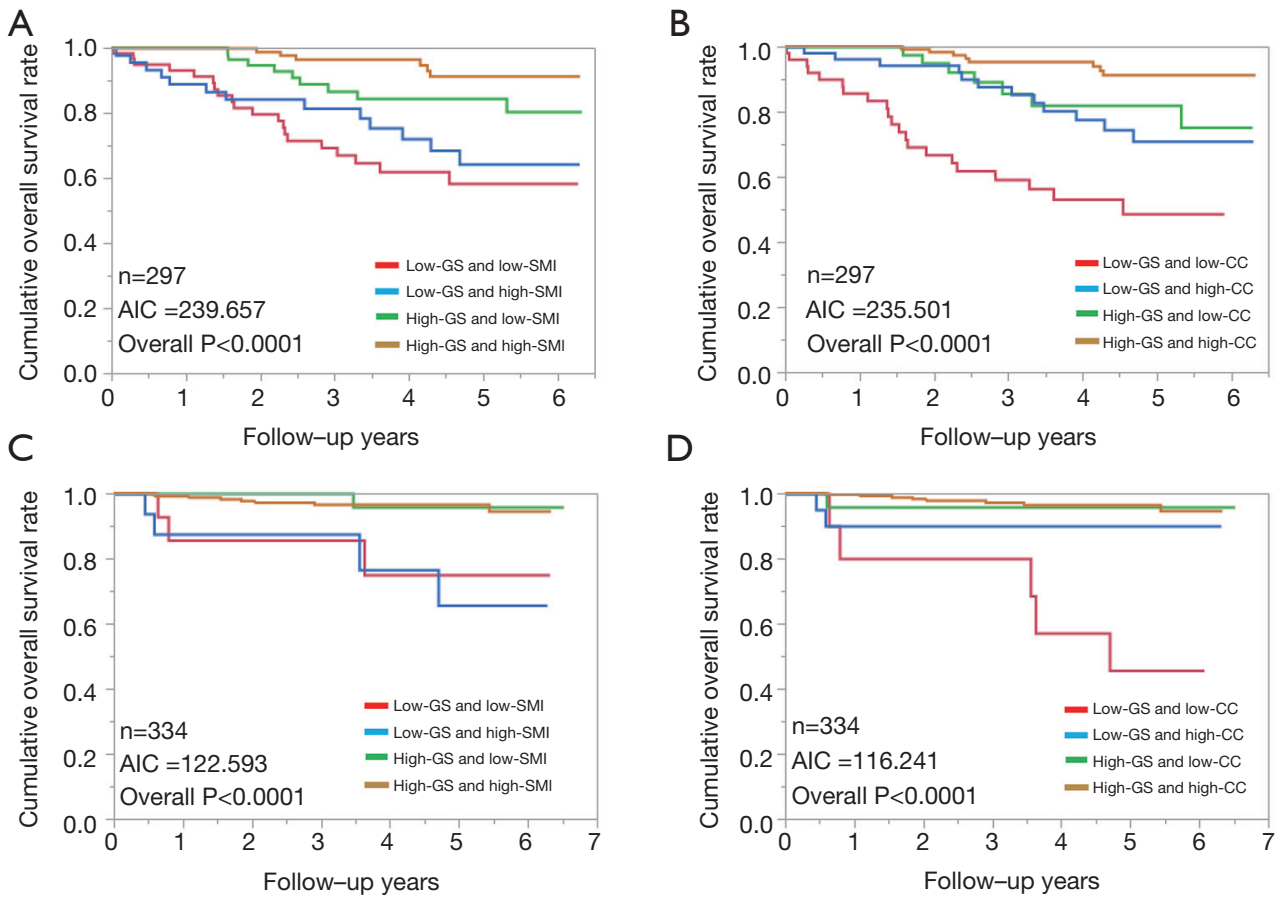

Figure 4 Mortality rates stratified by GS and SMI or CC according to age. (A) Mortality rates stratified by GS and SMI in patients over 65 years. (B) Mortality rates stratified by GS and CC in patients over 65 years. (C) Mortality rates stratified by GS and SMI in patients less than 65 years. (D) Mortality rates stratified by GS and CC in patients less than 65 years. GS, grip strength; SMI, skeletal muscle index; CC, calf circumference.
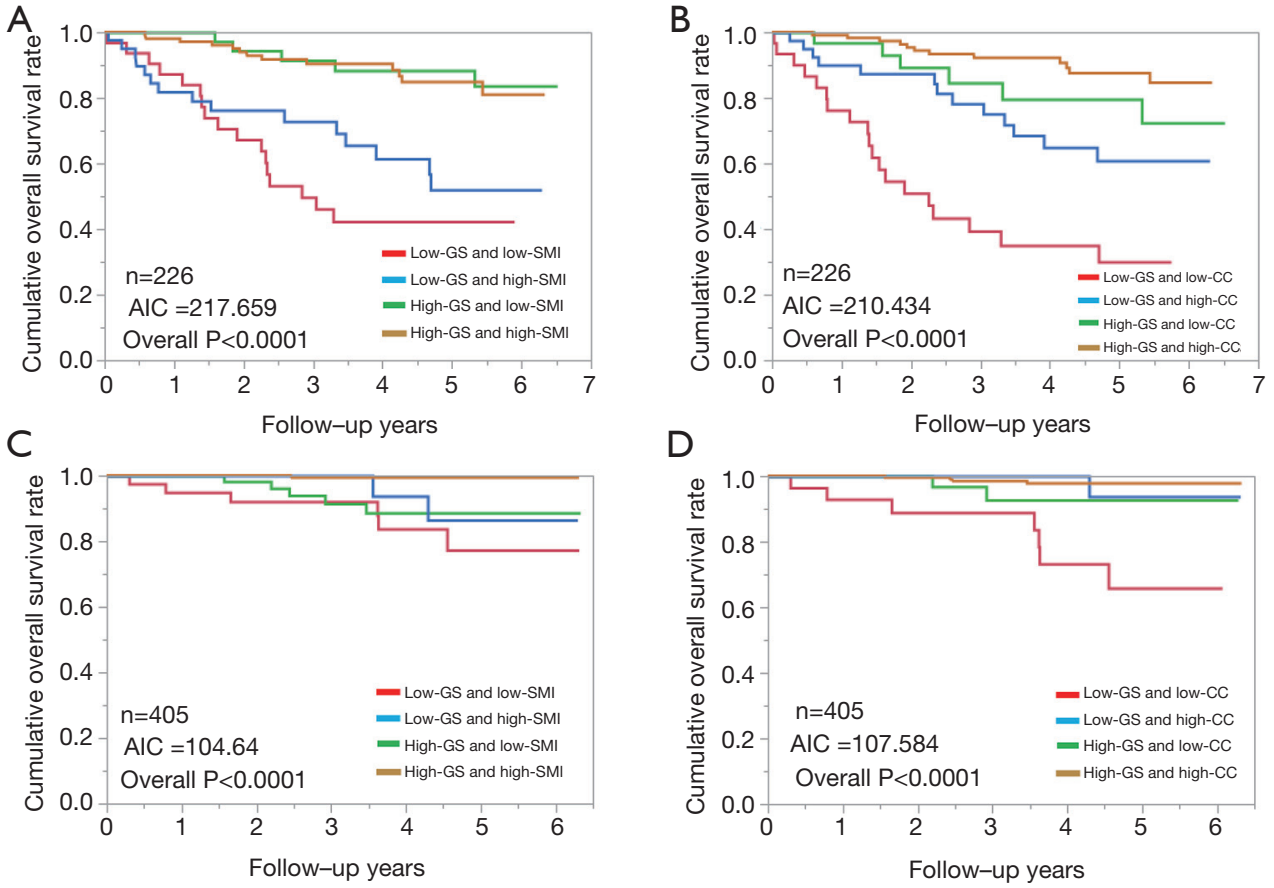

Figure 5 Mortality rates stratified by GS and SMI or CC according to the LC status. (A) Mortality rates stratified by GS and SMI in LC patients. (B) Mortality rates stratified by GS and CC in LC patients. (C) Mortality rates stratified by GS and SMI in non-LC patients. (D) Mortality rates stratified by GS and CC in non-LC patients. GS, grip strength; SMI, skeletal muscle index; CC, calf circumference; LC, liver cirrhosis. 
A

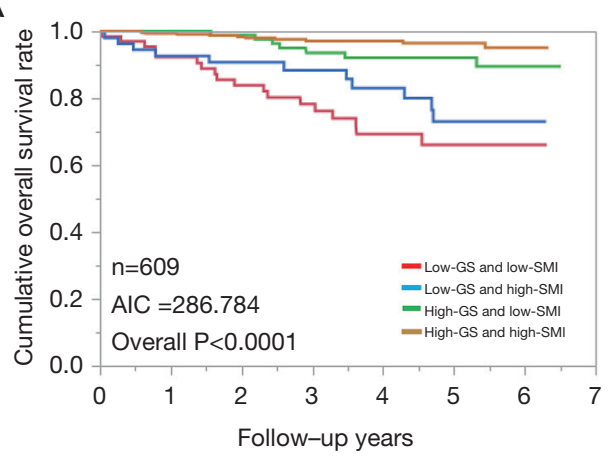

B

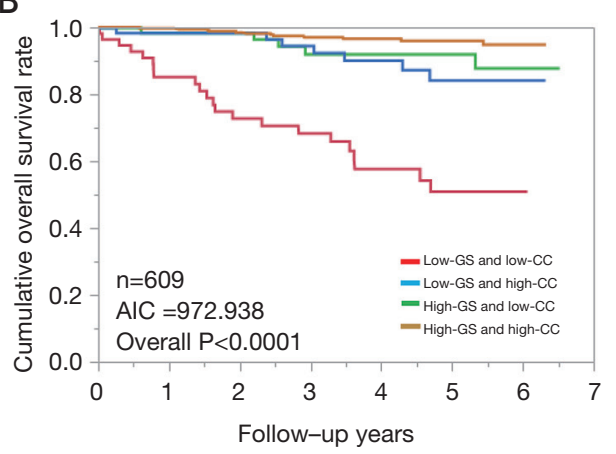

Figure 6 Mortality rates stratified by GS and SMI or CC in patients without HCC. (A) Mortality rates stratified by GS and SMI in patients without HCC. (B) Mortality rates stratified by GS and CC in patients without HCC. GS, grip strength; SMI, skeletal muscle index; CC, calf circumference; HCC, hepatocellular carcinoma.
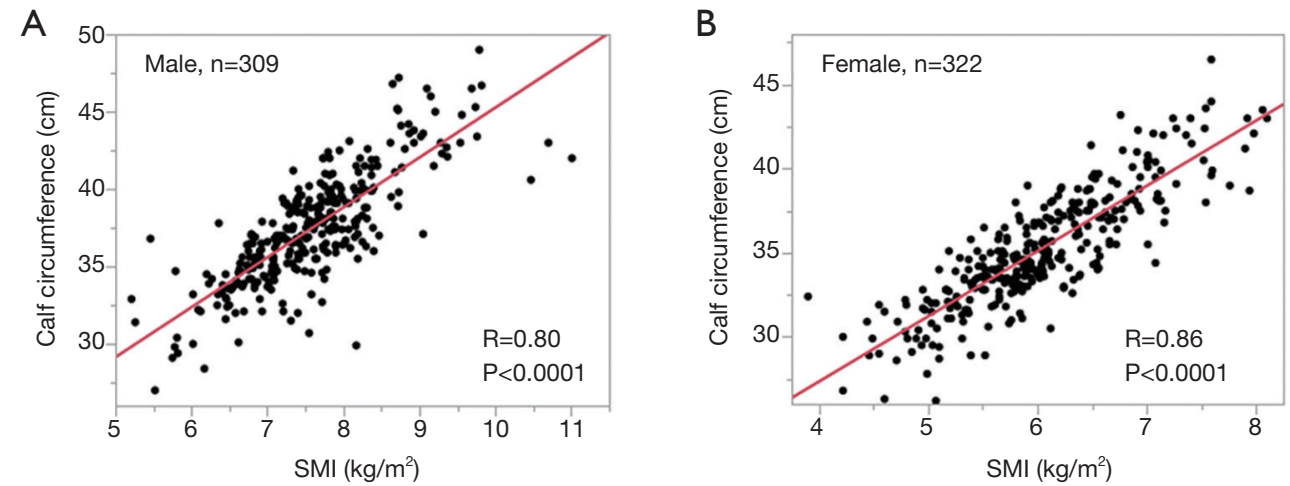

Figure 7 Correlation between CC and SMI in men (A) and women (B). CC, calf circumference; SMI, skeletal muscle index.
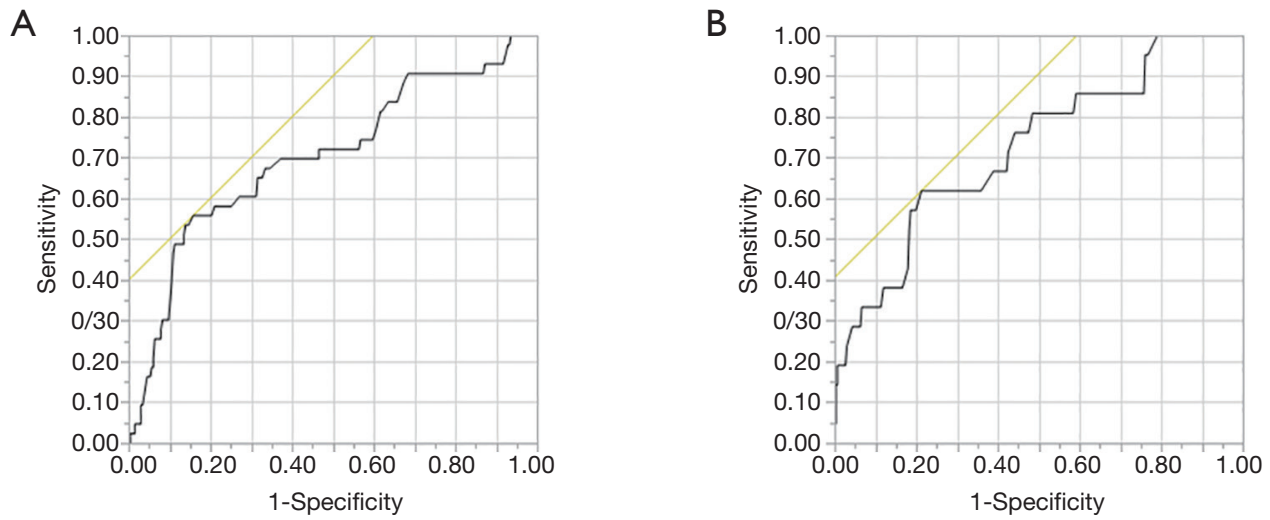

Figure 8 ROC analysis of calf circumference based on the overall survival in men (A) and women (B). ROC, receiver operating characteristic curve.

measure muscle mass, anthropometry measurements are of great significance due to its convenience for use. Different algorithms between the community situations and the hospital situations are presented in the revised Asian guidelines (22). In the community situations, characteristically, muscle mass measurement is not 
mandatory (22). Our study is probably the first report presenting the impact of CC on the OS in CLDs using a large cohort $(\mathrm{n}=631)$. Multiple results were presented, but we believe that all the results are clinically meaningful and worthy of reporting.

In our results, lower GS (hazard ratio $=6.110, \mathrm{P}<0.0001$ ) and lower $\mathrm{CC}$ (hazard ratio $=5.031, \mathrm{P}<0.0001$ ) were independent adverse factors contributing to the OS. Additionally, AIC values of combined GS-CC system were smaller than those of GS-SMI system for the entire cohort and all subgroups except for non-LC cases. These results denoted that our proposed combined GS-CC system can be a useful stratification system in CLD patients, which shed some lights on the daily clinical practice. On the other hand, skeletal muscle atrophy undoubtedly occurs with aging (11). However, in the multivariate analysis, advanced age was not a significant factor linked to the $\mathrm{OS}(\mathrm{P}=0.6400)$, while presence of LC (hazard ratio $=6.041, \mathrm{P}<0.0001$ ) and higher serum albumin level (hazard ratio $=0.474$, $\mathrm{P}=0.0355$ ) were significant as well as low-GS and low-CC. These results may be attributed to the impact of secondary sarcopenia caused by CLD itself on OS rather than ageassociated primary sarcopenia. A lot of previous reports demonstrated the prognostic significance of skeletal muscle mass as an independent predictor linked to the mortality, however, SMI was not significant in our multivariate analysis (2,34-40). Clinical impacts of GS and CC on the OS may diminish the impacts of SMI on the OS, but these results do not always deny the importance of skeletal muscle mass on the mortality in patients with CLDs. While the hazard ratio of GS on the OS was 6.110, which was higher than that of the presence of LC (6.041), also demonstrating strong impact of GS on the OS. Both CC and SMI are markers for skeletal muscle mass. Nevertheless, the HRs of CC and SMI on the OS were largely different (5.031 vs. 1.435). Reference values for low-CC and low-SMI may be linked to our results.

As expected, CC had a strong correlation with SMI both in men $(r=0.80)$ and women $(r=0.86)$ in our data. Kawakami et al. demonstrated that CC had a strong positive correlation with appendicular skeletal muscle and SMI in 526 adults (31). Hiraoka et al. reported the finger-circle test as an easy and effective screening method for early stage of muscle atrophy (41). All of these are in line with our current results.

Looking at Kaplan-Meier curves of combined GSSMI system and combined GS-CC system, we noticed a significant difference in the two systems. In comparison of type $\mathrm{A}$ and $\mathrm{B}$, the difference of OS did not reach significance, whereas in comparison of type $\mathrm{E}$ and $\mathrm{F}$, the difference of OS reached significance. Our proposed GSCC stratification system can identify the group with the worst prognosis. We would like to emphasize this point as the major strength of our proposed GS-CC stratification system compared to GS-SMI stratification system. In CLD patients with lower GS and lower CC, earlier intervention should be considered. While in our non-LC patients, 14 patients $(3.5 \%)$ died in the follow-up period. Of these, only one patient died of liver-related disease. One patient died of amyotrophic lateral sclerosis. The majority of causes of death in non-LC patients were cancer-related (not HCC) deaths. In our LC patients, 50 patients $(22.1 \%)$ died in the follow-up period. Of these, 44 patients died of liverrelated disease. In non-LC patients with adverse predictors (i.e., lower GS, lower SMI, or lower CC), the possibilities of diseases other than liver diseases should be taken into account. As described, AIC values of combined GS-CC system were smaller than those of GS-SMI system for all subgroups except for non-LC cases. This may be attributed to the difference of cause of death between non-LC patients and LC patients.

Male was an independent adverse predictor in our analysis. One possible reason for these is that the prevalence of HCC at baseline between male and female was significantly different $[5.2 \%(16 / 309) v s .1 .9 \%(6 / 322)$, $\mathrm{P}=0.0290]$. Decreased eGFR was also an independent adverse predictor. Several reports have stressed the prognostic significance of eGFR in CLD patients, which were in agreement with our results (42-44). While, BMI and WC (adiposity-related markers) were not associated with the OS in our study, although a recent large prospective cohort study demonstrated the prognostic significance of excess adiposity (45). The reason for these discrepancies between studies remains unclear. Antiviral therapies have dramatically improved in recent years $(46,47)$. The favorable hazard ratios in our patients with viral causes compared with non-viral causes seem to be attributable to the advancement of antiviral therapies. On the other hand, it is of note that in our ROC analysis of CC based on the OS, the optimal cutoff points were $34.6 \mathrm{~cm}$ in men and $32.8 \mathrm{~cm}$ in women, which were almost similar to the AWGS data (34 $\mathrm{cm}$ in men and $33 \mathrm{~cm}$ in women). Our results support the validity of the AWGS recommendations. Our study is the first study presenting the optimal cutoff point of CC in CLD patients using the OS data. This point should also be noted. 
Several limitations to our study should be mentioned. First, this single-center study had a retrospective nature. Second, the observation period (median, 3.53 years) may not be enough for the survival analysis especially in nonLC patients as the number of deaths in non-LC patients is expected to be small. Third, our cohort was heterogeneous including various etiologies for underlying liver diseases and various degrees of liver functional reserve. Fourth, the number of HCC patients was small $(\mathrm{n}=22)$, and thus whether our proposed prognostic system can be applied to HCC patients is unclear. Fifth, various interventions for each subject have been done during the follow-up period, making bias for the OS. Caution must be therefore taken in interpreting the results and external validation will be needed in future studies. Despite the limitations, the current study results denote that our proposed GS-CC prognostic system in patients with CLDs can be a useful stratification system. The usefulness of this system can be prominent in community settings without BIA or computed tomography. CC can be an alternative marker for SMI in patients with CLDs. In conclusion, we would like to emphasize the importance of GS-CC system as a useful prognostic model for patients with CLDs.

\section{Acknowledgments}

The authors would like to thank Yasuko Higuchi in our hospital for the anthropometry measurement. This work was partly supported by Hyogo Innovative Challenge, Hyogo College of Medicine, Japan.

Funding: None.

\section{Footnote}

Reporting Checklist: The authors have completed the STROBE reporting checklist. Available at http://dx.doi. org/10.21037/atm-20-6901

Data Sharing Statement: Available at http://dx.doi. org/10.21037/atm-20-6901

Peer Review File: Available at http://dx.doi.org/10.21037/ atm-20-6901

Conflicts of Interest: All authors have completed the ICMJE uniform disclosure form (available at http://dx.doi. org/10.21037/atm-20-6901). The authors have no conflicts of interest to declare.
Ethical Statement: The authors are accountable for all aspects of the work in ensuring that questions related to the accuracy or integrity of any part of the work are appropriately investigated and resolved. The study was conducted in accordance with the Declaration of Helsinki (as revised in 2013) with ethical approval from the institutional review board in our hospital (approval number 3469). An opt out method was employed considering the retrospective nature of this study.

Open Access Statement: This is an Open Access article distributed in accordance with the Creative Commons Attribution-NonCommercial-NoDerivs 4.0 International License (CC BY-NC-ND 4.0), which permits the noncommercial replication and distribution of the article with the strict proviso that no changes or edits are made and the original work is properly cited (including links to both the formal publication through the relevant DOI and the license). See: https://creativecommons.org/licenses/by-nc-nd/4.0/.

\section{References}

1. Rosenberg I. Summary comments: epidemiological and methodological problems in determining nutritional status of older persons. Am J Clin Nutr 1989;50:1231-3.

2. Nishikawa H, Enomoto H, Ishii A, et al. Elevated serum myostatin level is associated with worse survival in patients with liver cirrhosis. J Cachexia Sarcopenia Muscle 2017;8:915-25.

3. Kim G, Kang SH, Kim MY, et al. Prognostic value of sarcopenia in patients with liver cirrhosis: A systematic review and meta-analysis. PLoS One 2017;12:e0186990.

4. Meyer F, Valentini L. Disease-Related Malnutrition and Sarcopenia as Determinants of Clinical Outcome. Visc Med 2019;35:282-91.

5. Bunchorntavakul C, Reddy KR. Review article: malnutrition/sarcopenia and frailty in patients with cirrhosis. Aliment Pharmacol Ther 2020;51:64-77.

6. Aby ES, Saab S. Frailty, sarcopenia, and malnutrition in cirrhotic patients. Clin Liver Dis 2019;23:589-605.

7. Chang KV, Chen JD, Wu WT, et al. Is sarcopenia associated with hepatic encephalopathy in liver cirrhosis? A systematic review and meta-analysis. J Formos Med Assoc 2019;118:833-42.

8. van Vugt JL, Levolger S, de Bruin RW, et al. Systematic Review and Meta-Analysis of the Impact of Computed Tomography-Assessed Skeletal Muscle Mass on Outcome in Patients Awaiting or Undergoing Liver Transplantation. 
Am J Transplant 2016;16:2277-92.

9. Chang KV, Chen JD, Wu W'T, et al. Association between Loss of Skeletal Muscle Mass and Mortality and Tumor Recurrence in Hepatocellular Carcinoma: A Systematic Review and Meta-Analysis. Liver Cancer 2018;7:90-103.

10. Hsu CS, Kao JH. Sarcopenia and chronic liver diseases. Expert Rev Gastroenterol Hepatol 2018;12:1229-44.

11. Clark BC, Manini TM. What is dynapenia? Nutrition 2012;28:495-503.

12. Yoh K, Nishikawa H, Enomoto H, et al. Grip Strength: A Useful Marker for Composite Hepatic Events in Patients with Chronic Liver Diseases. Diagnostics (Basel) 2020;10:238.

13. Hanai T, Shiraki M, Imai K, et al. Reduced handgrip strength is predictive of poor survival among patients with liver cirrhosis: A sex-stratified analysis. Hepatol Res 2019;49:1414-26.

14. Norman K, Stobäus N, Gonzalez MC, et al. Hand grip strength: outcome predictor and marker of nutritional status. Clin Nutr 2011;30:135-42.

15. Bohannon RW. Hand-grip dynamometry predicts future outcomes in aging adults. J Geriatr Phys Ther 2008;31:3-10.

16. Knudsen AW, Naver A, Bisgaard K, et al. Nutrition impact symptoms, handgrip strength and nutritional risk in hospitalized patients with gastroenterological and liver diseases. Scand J Gastroenterol 2015;50:1191-8.

17. Leong DP, Teo KK, Rangarajan S, et al. Prognostic value of grip strength: findings from the Prospective Urban Rural Epidemiology (PURE) study. Lancet 2015;386:266-73.

18. García-Hermoso A, Ramírez-Vélez R, Peterson MD, et al. Handgrip and knee extension strength as predictors of cancer mortality: a systematic review and meta-analysis. Scand J Med Sci Sports 2018;28:1852-8.

19. Madden AM, Smith S. Body composition and morphological assessment of nutritional status in adults: A review of anthropometric variables. J Hum Nutr Diet 2016;29:7-25.

20. Tur JA, Bibiloni MDM. Anthropometry, Body Composition and Resting Energy Expenditure in Human. Nutrients 2019;11:E1891.

21. Utkualp N, Ercan I. Anthropometric Measurements Usage in Medical Sciences. Biomed Res Int 2015;2015:404261.

22. Chen LK, Woo J, Assantachai P, et al. Asian Working Group for Sarcopenia: 2019 Consensus Update on Sarcopenia Diagnosis and Treatment. J Am Med Dir Assoc 2020. pii: S1525-8610(19)30872-2.

23. Yamagishi $\mathrm{K}$, Iso $\mathrm{H}$. The criteria for metabolic syndrome and the national health screening and education system in
Japan. Epidemiol Health 2017;39:e2017003.

24. Fukui H, Saito H, Ueno Y, et al. Evidence-based clinical practice guidelines for liver cirrhosis 2015. J Gastroenterol 2016;51:629-50.

25. Kokudo N, Takemura N, Hasegawa K, et al. Clinical practice guidelines for hepatocellular carcinoma: The Japan Society of Hepatology 2017 (4th JSH-HCC guidelines) 2019 update. Hepatol Res 2019;49:1109-13.

26. European Association for the Study of the Liver. EASL Recommendations on Treatment of Hepatitis C 2018. J Hepatol 2018;69:461-511.

27. Drafting Committee for Hepatitis Management Guidelines, the Japan Society of Hepatology. Japan Society of Hepatology Guidelines for the Management of Hepatitis B Virus Infection:2019 update. Hepatol Res 2020;50:892-923.

28. Omata M, Cheng AL, Kokudo N, et al. Asia-Pacific clinical practice guidelines on the management of hepatocellular carcinoma: a 2017 update. Hepatol Int 2017;11:317-70.

29. European Association for the Study of the Liver. EASL Clinical Practice Guidelines: Management of hepatocellular carcinoma. J Hepatol 2018;69:182-236.

30. Nishikawa H, Shiraki M, Hiramatsu A, et al. Japan Society of Hepatology guidelines for sarcopenia in liver disease (1st edition): Recommendation from the working group for creation of sarcopenia assessment criteria. Hepatol Res 2016;46:951-63.

31. Kawakami R, Murakami H, Sanada K, et al. Calf circumference as a surrogate marker of muscle mass for diagnosing sarcopenia in Japanese men and women. Geriatr Gerontol Int 2015;15:969-76.

32. Akaike H. A New Look at the Statistical Model Identification. IEEE Transactions on Automatic Control AC 1974;19:716-23.

33. Akobeng AK. Understanding diagnostic tests 3: Receiver operating characteristic curves. Acta Paediatr 2007;96:644-7.

34. Nishikawa H, Enomoto H, Ishii A, et al. Prognostic significance of low skeletal muscle mass compared with protein-energy malnutrition in liver cirrhosis. Hepatol Res 2017;47:1042-52.

35. Kobayashi T, Kawai H, Nakano O, et al. Rapidly declining skeletal muscle mass predicts poor prognosis of hepatocellular carcinoma treated with transcatheter intraarterial therapies. BMC Cancer 2018;18:756.

36. Ebadi M, Wang CW, Lai JC, et al. Poor performance of psoas muscle index for identification of patients with higher waitlist mortality risk in cirrhosis. J Cachexia 
Sarcopenia Muscle 2018;9:1053-62.

37. Hamaguchi Y, Kaido T, Okumura S, et al. Preoperative Visceral Adiposity and Muscularity Predict Poor Outcomes after Hepatectomy for Hepatocellular Carcinoma. Liver Cancer 2019;8:92-109.

38. Ishizu Y, Ishigami M, Kuzuya T, et al. Low skeletal muscle mass predicts early mortality in cirrhotic patients with acute variceal bleeding. Nutrition 2017;42:87-91.

39. Montano-Loza AJ, Angulo P, Meza-Junco J, et al. Sarcopenic obesity and myosteatosis are associated with higher mortality in patients with cirrhosis. J Cachexia Sarcopenia Muscle 2016;7:126-35.

40. Fujiwara N, Nakagawa H, Kudo Y, et al. Sarcopenia, intramuscular fat deposition, and visceral adiposity independently predict the outcomes of hepatocellular carcinoma. J Hepatol 2015;63:131-40.

41. Hiraoka A, Izumoto H, Ueki H, et al. Easy surveillance of muscle volume decline in chronic liver disease patients using finger-circle (yubi-wakka) test. J Cachexia Sarcopenia Muscle 2019;10:347-54.

42. Asrani SK, Jennings LW, Kim WR, et al. MELD-GRAIL-

Cite this article as: Nishikawa $\mathrm{H}$, Yoh K, Enomoto H, Nishimura T, Nishiguchi S, Iijima H. Combined grip strength and calf circumference as a useful prognostic system in patients with liver diseases: a large cohort study. Ann Transl Med 2021;9(8):624. doi: 10.21037/atm-20-6901
Na: Glomerular Filtration Rate and Mortality on LiverTransplant Waiting List. Hepatology 2020;71:1766-74.

43. Allen AM, Kim WR, Therneau TM, et al. Chronic kidney disease and associated mortality after liver transplantation-a time-dependent analysis using measured glomerular filtration rate. J Hepatol 2014;61:286-92.

44. Cholongitas E, Arsos G, Goulis J, et al. Glomerular filtration rate is an independent factor of mortality in patients with decompensated cirrhosis. Hepatol Res 2014;44:E145-55.

45. Simon TG, Kim MN, Luo X, et al. Physical activity compared to adiposity and risk of liver-related mortality: Results from two prospective, nationwide cohorts. J Hepatol 2020;72:1062-9.

46. Spearman CW, Dusheiko GM, Hellard M, et al. Hepatitis C. Lancet 2019;394:1451-66.

47. Sarin SK, Kumar M, Eslam M, et al. Liver diseases in the Asia-Pacific region: a Lancet Gastroenterology \& Hepatology Commission. Lancet Gastroenterol Hepatol 2020;5:167-228. 